\title{
CONCERNING WEBS IN THE PLANE
}

\author{
BY \\ MARY-ELIZABETH HAMSTROM
}

1. Introduction. A compact continuum $M$ is said( $\left.{ }^{1}\right)$ to be a web if and only if there exist two upper semicontinuous collections $G_{1}$ and $G_{2}$ such that (1) each of these collections fills up $M$, (2) each of them is a dendron with respect to its elements, and (3) there exists an uncountable subcollection $W$ of the collection $G_{1}$ such that no element of $W$ is a subset of any element of $G_{2}$. If there exist two collections $G_{1}$ and $G_{2}$ satisfying all of the above conditions together with the additional condition that every element of $G_{1}$ intersects each element of $G_{2}$ in a totally disconnected point set, then the web $M$ is said $\left({ }^{2}\right)$ to be simple.

In the above definition of a web "dendron" may be replaced( $\left.{ }^{3}\right)$ by "arc." But R. H. Bing has shown (4) by an example that a corresponding change in the definition of simple web would alter the meaning of that term.

In the present paper the notions of a $W_{n}$ set and a $W_{n}^{\prime}$ set are introduced.

DEFINITION. If $n>1, a W_{n}$ set is a compact continuum $M$ for which there exists a family $F$ of $n$ elements such that (1) each element of $F$ is an upper semicontinuous collection of mutually exclusive continua which fills up $M$ and is an arc with respect to its elements, (2) if $G$ is a collection of continua each belonging to some, but no two to the same, collection of the family $F$, then the continua of the collection $G$ have a point in common and their common part is totally disconnected.

Definition. $A W_{n}^{\prime}$ set is a $W_{n}$ set satisfying the conditions obtained by replacing, in the above definition, the phrase "upper semicontinuous collection of mutually exclusive continua" by the phrase "continuous collection of mutually exclusive continuous curves."

Hereafter in this paper the space considered will be the Euclidean plane. It will be shown that there exists a $W_{2}$ set which is not a $W_{3}$ set and that for each positive integer $n$ a simple closed curve plus its interior is a $W_{n}$ set. A necessary and sufficient condition for a compact continuum to be a $W_{2}^{\prime}$ set will also be established. I wish to express my deep appreciation to Professor

Presented to the Society, September 5, 1952, the theorems of $\$ 7$ were presented under the title Concerning a certain type of web; received by the editors June 9, 1952 and, in revised form, August 11, 1952.

(1) R. L. Moore, Concerning continua which have dendratomic subsets, Proc. Nat. Acad. Sci. U. S. A. vol. 29 (1943) pp. 384-389.

(2) R. L. Moore, A characterization of a simple plane web, Proc. Nat. Acad. Sci. U. S. A. vol. 32 (1946) pp. 311-316; and R. H. Bing, Concerning simple plane webs, Trans. Amer. Math. Soc. vol. 60 (1946) pp. 133-148.

(3) Cf. Theorem 1 of paper cited in footnote 1.

(4) R. H. Bing, loc. cit. 
R. L. Moore who aroused my interest in these problems and whose many helpful suggestions were an invaluable aid in the preparation of this paper.

2. Filling up a compact continuous curve whose boundary is the sum of two mutually exclusive simple closed curves.

THEOREM 1. If $M$ is the compact continuum whose boundary is the sum of two circles $C_{1}$ and $C_{2}$ with center at the origin and radii 1 and 2 respectively, then there exists a family $F$ of $n$ elements such that (1) each element of $F$ is a continuous collection of mutually exclusive arcs which fills up $M$ and is a simple closed curve with respect to its elements, (2) if $G$ is a collection of arcs each belonging to some, but no two to the same, collection of the family $F$, then the arcs of the collection $G$ have a point in common and their common part is totally disconnected.

Proof. We shall begin by proving the theorem in detail for the case $n=3$. Let $S$ be the square plus its interior with vertices $(0,0),(0,1),(1,0)$, and $(1,1)$. Hereafter, when referring to $S$ we shall use rectangular co-ordinates; when referring to $M$, polar co-ordinates. Let $I$ be an interval of length 1 with end points $A$ and $B$.

For each non-negative number $a$ not greater than 1 , let $m_{a}$ be the collection of all points in $S$ with ordinate $a$ and let $k_{a}$ be the collection of all those with abscissa $a$. For each non-negative number $a$ not greater than 2 , let $r_{a}$ be the collection of all points $(r, \theta)$ in $M$ such that $\theta=2 \pi a$.

There exists a continuous transformation $T$ throwing $I$ into $S$ such that if the subset $K$ of $I$ contains a domain with respect to $I, T(K)$ contains a domain with respect to $S$ and $T(A)$ and $T(B)$ are vertices of $S$.

For each non-negative number $z$ less than 1 let $T_{1 z}$ denote a transformation of $I$ into a subset $I_{1 z}$ of $M$ such that if $P$ is a point of $I$ such that $A P=x$ and if $T(P)$ is a point of $k_{y}$, then $T_{1 z}(P)=(1+x, 2 \pi(y+z))$. In $M, T_{1 z}(P)$ is a point of the interval $r_{y+z}$ at a distance $x$ from $C_{1}$.

For each $z, T_{1 z}$ is reversibly continuous and therefore $I_{1 z}$ is an arc. For each point $P$ of $M$ there is only one number $z$ such that $P$ belongs to $I_{1 z}$. Let $G_{1}$ be the collection of all $I_{1 z}$ 's. Since, for each $z, I_{1 z}$ can be obtained from $I_{10}$ by means of a rotation about the origin, the collection $G_{1}$ is continuous. So it is a continuous collection of mutually exclusive arcs, it fills up $M$, and it is a simple closed curve with respect to its elements.

If $z$ and $z^{\prime}$ are non-negative numbers less than 1 and $C$ is the set of all points $P$ of $I$ such that $T_{1 z}(P)$ is a point of $r_{z^{\prime}}$, then, depending on whether or not $z^{\prime}-z$ is non-negative, $T(C)$ is either $k_{z^{\prime}-z}$ or $k_{z^{\prime}-z+1}$ and is therefore uncountable. But no interval contains a domain. Therefore $C$ contains no segment and consequently the common part of $I_{1 z}$ and $r_{z^{\prime}}$ is uncountable and totally disconnected.

Now, for each non-negative number $z^{\prime}$ less than 1 let $T_{2 z^{\prime}}$ denote a transformation of $I$ into a subset $I_{2 z^{\prime}}$ of $M$ such that if $P^{\prime}$ is a point of $I$ such 
that $A P^{\prime}=x^{\prime}$ and $T\left(P^{\prime}\right)$ belongs to $m_{y^{\prime}}$, then $T_{2 z^{\prime}}\left(P^{\prime}\right)$ is a point of the interval $r_{y^{\prime}+z^{\prime}}$ at a distance $x^{\prime}$ from $C_{1}$. Let $G_{2}$ be the collection of all $I_{2 z^{\prime}}$ 's. Then $G_{2}$ is a continuous collection of mutually exclusive arcs filling up $M$ and it is a simple closed curve with respect to its elements. Also, if $z$ and $z^{\prime}$ are nonnegative numbers less than 1 , the common part of $I_{2 z^{\prime}}$ and $r_{z}$ exists and is totally disconnected.

Let $z, z^{\prime}$, and $y$ each denote a non-negative number less than 1 . We shall prove that $I_{1 z}, I_{2 z^{\prime}}$, and $r_{y}$ have a common part. Let $P^{\prime}$ be the point of intersection of $k_{y-z}$ (or $k_{y-z+1}$ if $y<z$ ) and $m_{y-z^{\prime}}\left(\right.$ or $m_{y-z^{\prime}+1}$ if $y<z^{\prime}$ ), and let $P$ be a point of $I$ such that $T(P)=P^{\prime}$. If $x$ is the distance from $P$ to $A, T_{1 z}(P)$ is on $r_{y}$ at a distance $x$ from $C_{1}$ and $T_{2 z^{\prime}}(P)$ is on $r_{y}$ at a distance $x$ from $C_{1}$. Therefore $T_{1 z}(P)=T_{2 z^{\prime}}(P)$.

We now prove that the common part of $I_{1 z}$ and $I_{2 z^{\prime}}$ is totally disconnected $\left({ }^{5}\right)$. Let $\alpha$ be an arc in $M$ with end points $\left(r_{1}, \theta_{1}\right)$ and $\left(r_{2}, \theta_{2}\right)$ where $r_{1}<r_{2}$. Since no subinterval of $I$ goes into an arc under $T$, there is a point $P$ of $I$ such that $r_{1}<A P+1<r_{2}$ and $[z+\operatorname{abscissa} T(P)]-\left[z^{\prime}+\right.$ ordinate $\left.T(P)\right]$ is not an integer. Consequently $T_{1 z}(P)$ and $T_{2 z^{\prime}}(P)$ do not have the same $\theta$ co-ordinate and the point of $\alpha$ at distance $A P$ from $C_{1}$ is not a point of the common part of $I_{1 z}$ and $I_{2 z^{\prime}}$. Since this common part does not contain an arc, it is totally disconnected.

Let $G_{3}$ be the collection of all $r_{y}$ 's. The collections $G_{1}, G_{2}$, and $G_{3}$ satisfy the conditions of Theorem 1 .

If $k$ is any positive integer, the preceding arguments may be easily extended to prove Theorem 1 for the case $n=k$. We need only let $S$ be the $(k-1)$-dimensional cube consisting of all points whose rectangular coordinates are non-negative numbers not greater than 1 and consider, in place of the intervals $k_{a}$ and $m_{a}$, the intersections of $S$ and an appropriate selection of $k-1$ planes.

It is also possible to obtain a family of infinitely many elements which satisfies the conditions of Theorem 1 . Let $S$ be the collection of all points which are infinite simple sequences each element of which is a non-negative number not greater than 1 . The sequence $P_{1}, P_{2}, \cdots$ is said to converge to the point $P$ if and only if for each positive integer $n$ the $n$th elements of the sequences $P_{1}, P_{2}, \cdots$ converge to the $n$th element of $P$. The set $S$ is seen to be a compact continuous curve and therefore the preceding arguments may be extended to this case.

\section{Filling up a simple closed curve plus its interior.}

TheOREM 2. If $M^{\prime}$ is a simple closed curve plus its interior and $n$ is a positive integer, then $M^{\prime}$ is a $W_{n}$ set.

Proof. Let $C_{1}, C_{2}$, and $M$ be as defined in the proof of Theorem 1. It fol-

(5) The author is indebted to the referee, who suggested ways of shortening the argument here and of shortening the arguments in the proofs of Theorems 2,11, and 12. 
lows from the proof of Theorem 1 that if $n$ is a positive integer, there exist $n$ collections $G_{1}, G_{2}, \cdots, G_{n}$ each filling up $M$ and satisfying the conditions of Theorem 1 such that for each $i$ each arc of $G_{i}$ has one end point on $C_{1}$ and one end point on $C_{2}$, these points having the same $\theta$ co-ordinate, and each point of $C_{1}+C_{2}$ is an end point of some arc of $G_{i}$.

There is an upper semicontinuous collection $G$ of mutually exclusive point sets filling up $M$ such that each element of $G$ is either a point of $M-C_{1}$ or the common part of $C_{1}$ and some vertical line. For each $i$ and each nonnegative number $x$ not greater than $\pi$ let $h_{i x}$ denote the subcollection of $G$ the sum of whose elements is the sum of the elements of $G_{i}$ which contain either $(1, x)$ or $(1,-x)$. The collection $h_{i x}$ is an arc with respect to its elements, and $H_{i}$, the collection of all $h_{i x}$ 's, is an arc with respect to its elements. The family $H_{1}, H_{2}, \cdots, H_{n}$ satisfies the conditions of Theorem 2 with respect to the elements of the collection $G$ which, with respect to its elements, is topologically equivalent to $M^{\prime}$.

It follows from the proof of Theorem 2 and the concluding remarks in the proof of Theorem 1 that there exists a family of infinitely many collections which satisfies conditions similar to those required for a $W_{n}$ set with respect to $M^{\prime}$.

\section{4. $W_{1}$ sets and their boundaries.}

Definition. $A W_{1}$ set is a compact continuous curve $M$ such that (1) every complementary domain of $M$ is bounded by a simple closed curve which has no more than one point in common with the boundary of any other complementary domain of $M$, and (2) there exists an upper semicontinuous collection of mutually exclusive continua which fills $u p M$ and is an arc with respect to its elements.

Throughout this paper $E$ will denote the set of all points in the plane. If $K$ is a subcontinuum of the $W_{1}$ set $M$, the notation $M_{K}$ will be used to denote a point set such that $P$ is a point of it if and only if $P$ is a point either of $K$ or of some complementary domain of $M$ whose boundary is a subset of $K$.

The following theorem holds true. It is clear that it and Theorem 4 remain true under much less restrictive hypotheses.

THEOREM 3. If $K$ is a subcontinuum of the $W_{1}$ set $M$, then if $M_{K}$ separates $E, K$ separates both $E$ and $M$ and if $M-K$ is the sum of two mutually separated connected point sets $L$ and $N$ and $M_{K}$ separates $E$, then $E-M_{K}$ is the sum of two mutually separated connected point sets of which one contains $L$ and the other contains $N$.

THEOREM 4. If the subcontinuum $K$ of the $W_{1}$ set $M$ separates $M$ and $M_{K}$ does not separate $E$, then the common part of $K$ and the boundary of some complementary domain of $M$ exists and is not connected.

Proof. There exists a complementary domain of $M$ whose boundary contains a point of $M-K$. Otherwise $M-K$ would be identical with $E-M_{K}$ 
contrary to the supposition that $K$ separates $M$ and $M_{K}$ does not separate $E$.

Let $D_{1}, D_{2}, \cdots$, be the complementary domains of $M$ whose boundaries intersect $M-K$. Let $J_{1}, J_{2}, \cdots$, be their respective boundaries. By hypothesis $M-K$ is the sum of two mutually separated point sets $L$ and $N$. Suppose that for each $i$ either $J_{i}$ does not contain a point of $K$ or $K \cdot J_{i}$, and consequently $J_{i}-K \cdot J_{i}$, is connected. Let $K_{i}$ be the set of all points of $J_{i}$ that do not belong to $K$. For each $i, K_{i}$ is connected and is therefore a subset either of $L$ or of $N$. Let $N_{D}$ be the sum of all the $D_{i}$ 's such that $K_{i}$ is a subset of $N$ and let $L_{D}$ be the sum of all others. The point set $E-M_{K}$ is the sum of the mutually separated point sets $L+L_{D}$ and $N+N_{D}$, contrary to the hypothesis.

Suppose $M$ is a $W_{1}$ set and $G$ is an upper semicontinuous collection of mutually exclusive continua which fills up $M$ and is an arc with respect to its elements. Then the following Theorems 5-9 hold true.

THEOREM 5. If $g$ is an end element of $G$, then there do not exist two complementary domains of $M$ each bounded by a simple closed curve which intersects $g$ but does not lie wholly in it.

Proof. Suppose there do exist two such complementary domains $D_{1}$ and $D_{2}$ with boundaries $J_{1}$ and $J_{2}$ respectively. Since the continuum $g$ does not separate $M, J_{1} \cdot g$ and $J_{2} \cdot g$ are connected. Let $t_{1}$ and $t_{2}$ be arcs each of which lies except for one end point in $J_{1}-J_{1} \cdot g$ and does not contain a point of the common part of the other one and $J_{1}-J_{1} \cdot g$. Let $t_{3}$ and $t_{4}$ be two such arcs in $J_{2}$.

For each $i$ let $H_{i}$ denote the collection of all elements of $G$ which intersect $t_{i}$. The collection $H_{i}$ is an arc with respect to its elements and $g$ is an end element of this arc. Since $g$ is also an end element of $G$, the common part of the collections $H_{1}, H_{2}, H_{3}$, and $H_{4}$ is a collection $H^{\prime}$ which is an arc with respect to its elements. Every element of $H^{\prime}$ other than $g$ intersects each $t_{i}-t_{i} \cdot g$. Moreover, there is an element, $h$, of $H^{\prime}$ distinct from $g$ which does not contain any point of $J_{1}+J_{2}$ not in one of the $t_{i}$ 's. Neither of the point sets $h \cdot J_{1}$ and $h \cdot J_{2}$ is connected. Consequently there are points $P_{1}$ and $P_{2}$ of $J_{1}$ and $J_{2}$ respectively such that $h$ separates $g$ from $P_{1}$ in $E-D_{1}$ and $g$ from $P_{2}$ in $E-D_{2}$. But $D_{2}$ is a subset of $E-D_{1}$ and since $g$ intersects $J_{2}, h$ separates $g+P_{2}$ from $P_{1}$ in $E-D_{1}$. But $h, g, P_{1}$, and $P_{2}$ are subsets of $E-\left(D_{1}+D_{2}\right)$ and $M$. Consequently $h$ separates $g$ from $P_{1}, P_{1}$ from $P_{2}$, and $P_{2}$ from $g$ in $M$, which is impossible, since $M-h$ is the sum of two mutually separated connected point sets. Therefore the supposition that there exist domains such as $D_{1}$ and $D_{2}$ is false.

Theorem 6. Suppose $g$ is an element of $G$ which separates $M$. If $M_{g}$ does not separate $E$ there do not exist two complementary domains of $M$ each bounded by a simple closed curve which intersects $g$ but does not lie wholly in it, and if $M_{o}$ does separate $E$ there do not exist three such domains. 
Proof. If $M_{0}$ does not separate $E$, then by Theorem 4 there exists a simple closed curve $J_{1}$ bounding some complementary domain of $M$ and such that $J_{1} \cdot g$ exists and is not connected. Suppose there exists a simple closed curve $J_{2}$, different from $J_{1}$, which is the boundary of a complementary domain of $M$ and intersects, but is not a subset of, $g$. The common part of $J_{2}$ and $g$ is connected. Otherwise it would follow from the proof of Theorem 5 that $M-g$ is the sum of three mutually separated point sets, which is impossible.

The point set $M-g$ is the sum of two mutually separated connected point sets $L$ and $N$. The point set $J_{2}-J_{2} \cdot g$ is conrlected and therefore is a subset either of $L$ or of $N$. Suppose it is a subset of $L$. Since $J_{1} \cdot g$ is not connected, there exist two points of $J_{1}$ which are separated in $M$ by $g$ and since $L$ and $N$ are connected one of these points belongs to $L$ and the other to $N$. Therefore there exists an arc $t$ on $J_{1}$ which lies wholly in $L$ except for its end points, which belong to $g$, and there exist two nonintersecting arcs $t_{1}$ and $t_{2}$ each of which is a subset of $t$ and has an end point in $g$. Also, there exist two arcs $t_{3}$ and $t_{4}$ on $J_{2}$ such that each of them lies except for one end point in $J_{2}-J_{2} \cdot g$ and contains no point of the other one not in $g$. The argument of Theorem 5 may now be used to prove that $M-g$ is the sum of three mutually separated point sets. But this involves a contradiction.

Suppose $M_{g}$ does separate $E$ and that there exist three simple closed curves $J_{1}, J_{2}$, and $J_{3}$, each of which bounds a complementary domain of $M$ and intersects $g$ but is not a subset of it. For each $i$ let $t_{i}$ be an arc on $J_{i}$ which lies except for its end points in $J_{i}-J_{i} \cdot g$, or, if $J_{i} \cdot g$ consists of only one point, let $t_{i}$ be $J_{i}$. Each of the point sets $t_{1}-t_{1} \cdot g, t_{2}-t_{2} \cdot g$, and $t_{3}-t_{3} \cdot g$ is connected and is a subset either of $L$ or of $N$. Two of these are either both subsets of $L$ or both subsets of $N$. The argument of Theorem 5 may now be used to prove that $L$ is not connected. This is a contradiction.

Theorem 7. If $g$ is an element of $G$ and $M_{0}$ separates $E$, then (1) if $J_{1}$ is the boundary of a complementary domain of $M$ and intersects $g$, their common part is connected and (2) if $J_{1}$ is not a subset of $g$ and $J_{2}$ is the boundary of a complementary domain of $M$, is different from $J_{1}$, and intersects $g$ but is not a subset of it, then $J_{1}-J_{1} \cdot g$ and $J_{2}-J_{2} \cdot g$ are separated in $M$ by $g$.

Proof. It follows from Theorem 3 that $M-g$ is the sum of two mutually separated connected point sets $L$ and $N$. It also follows from Theorem 3 that $E-M_{0}$ is the sum of two mutually separated connected point sets $L^{\prime}$ and $N^{\prime}$ containing $L$ and $N$ respectively.

Let $D_{1}$ be the complementary domain of $M$ whose boundary is $J_{1}$ and su'ppose that $J_{1} \cdot g$ exists and is not connected. Since $J_{1}$ is not a subset of $g, E-M_{0}$ contains $D_{1}$ and $D_{1}$ is a subset either of $L^{\prime}$ or $N^{\prime}$-say $L^{\prime}$. Consequently $J_{1}-J_{1} \cdot g$ is a subset of $L^{\prime}$ and of $L$, contrary to the fact that $g$ separates two points of $J_{1}$ in $M$. Therefore $J_{1} \cdot g$ is connected. 
If neither of $J_{1}$ and $J_{2}$ is a subset of $g$, the point sets $J_{1}-J_{1} \cdot g$ and $J_{2}$ $-J_{2} \cdot g$ are connected. It follows from the proof of Theorem 6 that $g$ separates these point sets in $M$ and from Theorem 3 that $M_{g}$ separates them in $E$. Consequently $g$ separates $J_{1}-J_{1} \cdot g$ from $J_{2}-J_{2} \cdot g$ in $E$.

THEOREM 8. Suppose $J$, the boundary of some complementary domain of $M$, is not a subset of any element of $G$. Let $H$ be the collection of all elements of $G$ that intersect $J$. Then (1) there exist two elements $h$ and $h^{\prime}$ of $H$ such that $h \cdot J$ and $h^{\prime} \cdot J$ are connected and (2) if $g$ is an element of $H$ other than $h$ or $h^{\prime}, M_{g}$ does not separate $E$ and $J \cdot g$ is not connected.

Proof. The collection $H$ is an arc with respect to its elements. Let $h$ and $h^{\prime}$ denote its end elements. Suppose $h \cdot J$ is not connected. There exist two points $A$ and $B$ of $J$ that are separated by $h$ in $M$. The elements of $H$ that contain $A$ and $B$ are separated by $h$ in $M$ and consequently in $\left.H^{*}{ }^{6}\right)$. This is impossible. Hence $J \cdot h$ and $J \cdot h^{\prime}$ are connected.

By Theorems 3, 4, and 6, if $h$ is not an end element of $G$ (and consequently separates $M$ ), then $h$ separates $E$.

Let $g$ be an element of $H$ other than $h$ or $h^{\prime}$. The point set $g$ separates $h$ from $h^{\prime}$ in $M$. Therefore $J \cdot g$ separates $J \cdot h$ from $J \cdot h^{\prime}$ in $J$, which is possible only if $J \cdot g$ is not connected. It follows from Theorem 7 that $M_{\theta}$ does not separate $E$.

COROLlaRY. If $J$, the boundary of some complementary domain of $M$, is not a subset of any element of $G, H$ denotes the collection of all elements of $G$ that intersect $J, h$ and $h^{\prime}$ denote the end elements of $H$, and $J^{\prime}$ is the boundary of a complementary domain of $M$, is different from $J$, and intersects $H^{*}-\left(h+h^{\prime}\right)$, then $J^{\prime}$ is a subset of some element of $H$.

Proof. The simple closed curve, $J^{\prime}$, intersects some element $g$ of $H$ different from $h$ or $h^{\prime}$. The point set $J \cdot g$ is not connected, so $M_{g}$ does not separate $E$. It follows from Theorem 6 that $J^{\prime}$ is a subset of $g$.

THEOREM 9. Let $\alpha$ be a sequence of boundaries of distinct complementary domains of $M$ no term of which is a subset of any element of $G$. If the limiting set of $\alpha$ is a nondegenerate continuum, it is a subset of some element of $G$.

Proof. Let $K$ denote the limiting set of $\alpha$. Suppose $K$ intersects two elements, $g_{1}$ and $g_{2}$, of $G$. Let $S$ denote the sum of all elements of $G$ between $g_{1}$ and $g_{2}$. Since $K$ is connected, it intersects $S$ and consequently some element, $J$, of $\alpha$ intersects $S$. Since $J$ is not a subset of any element of $G$, it follows from Theorem 8 that there is an element, $g$, of $G$ between $g_{1}$ and $g_{2}$ such that $J \cdot g$ exists and is not connected. The point set $M-g$ is the sum of two mutually separated connected point sets $L$ and $N$. There exist two points of $J$

(6) If $H$ is a collection of mutually exclusive point sets the notation $H^{*}$ is used to denote the sum of the elements of $H$. 
that are separated by $g$ in $M$. Hence $L$ and $N$ both intersect $J$. The point sets $J \cdot L$ and $J \cdot N$ are both connected, for otherwise $g$ would separate $M$ into three mutually separated point sets.

Suppose $K$ is not a subset of $g$. Then it intersects either $L$ or $N$-say $L$. If $H_{1}$ denotes the collection of all elements of $G$ that intersect $L+g, H_{1}$ is an arc with respect to its elements and $g$ is an end element of this arc. If $H_{2}$ denotes $g$ plus the collection of all elements of $G$ that intersect $J \cdot L, H_{2}$ is an arc with respect to its elements, $g$ is an end element of this arc, and $H_{2}$ is a subcollection of $H_{1}$. Since $H_{2}^{*} \cdot J$ is connected, there is an element $h$ of $H_{2}$ such that $h \cdot J$ is connected and $h$ is an end element of $H_{2}$. Let $S^{\prime}$ denote the sum of all the elements of $H_{2}$ between $g$ and $h$. Since $K$ is connected, it intersects $S^{\prime}$. Hence, since $K$ is the limiting set of $\alpha$, there is an element $J^{\prime}$ of $\alpha$ which intersects $S^{\prime}-S^{\prime} . J$. There is an element, $h^{\prime}$, of $H_{2}$ between $h$ and $g$ which intersects $J^{\prime}$. But, by Theorem $8, J \cdot h^{\prime}$ is not connected and $M_{h^{\prime}}$ does not separate $E$. Therefore, by Theorem $6, J^{\prime}$ is a subset of $h^{\prime}$, contrary to hypothesis. Hence $K$ does not intersect two elements of $G$ and is therefore a subset of some element of $G$.

\section{Boundaries of $W_{3}^{\prime}$ sets.}

THEOREM 10. The limiting set of any infinite sequence of distinct boundaries of complementary domains of a $W_{3}$ set is totally disconnected.

Proof. Suppose $\alpha$ is an infinite sequence of distinct boundaries of complementary domains of the $W_{3}$ set $M$. The continuum $M$ is a simple web and therefore a continuous curve $\left({ }^{7}\right)$. Bing has shown $\left({ }^{8}\right)$ that each complementary domain of a simple web is bounded by a simple closed curve and that no two such boundaries have more than one point in common. Consequently $M$ is a $W_{1}$ set.

Let $G_{1}, G_{2}$, and $G_{3}$ be collections satisfying with respect to $M$ the requirements of the definition of a $W_{3}$ set. Suppose $K$, the limiting set of $\alpha$, contains a nondegenerate continuum $L$. The continuum $L$ is the limiting set of a subsequence $\alpha^{\prime}$ of $\alpha$.

Suppose that there exist infinitely many terms of $\alpha^{\prime}$ each lying in some element of $G_{1}$. Let $\alpha_{1}$ denote the subsequence of $\alpha^{\prime}$ whose terms are the terms of $\alpha^{\prime}$ having this property. Suppose that the limiting set of $\alpha_{1}$ contains a nondegenerate continuum $K^{\prime}$. The continuum $K^{\prime}$ is the limiting set of some subsequence $\alpha_{1}^{\prime}$ of $\alpha_{1}$. No element of $G_{2}$ or $G_{3}$ contains an element of $\alpha_{1}^{\prime}$. Therefore, by Theorem $9, K^{\prime}$ is a subset of an element of $G_{2}$ and of an element of $G_{3}$. Since $K^{\prime}$ is nondegenerate and connected, this is impossible.

Similarly there are not infinitely many elements of $\alpha^{\prime}$ each lying in some element of $G_{2}$ or $G_{3}$, and such that the limiting set of a sequence whose terms

(7) R. L. Moore, Concerning webs in the plane, Proc. Nat. Acad. Sci. U. S. A. vol. 29 (1943) pp. 389-393.

(8) R. H. Bing, loc. cit., Theorem 2. 
are these elements contains a nondegenerate continuum. Hence the continuum $L$ is the limiting set of the subsequence of $\alpha^{\prime}$ whose terms are the terms of $\alpha^{\prime}$ that do not lie in any element of $G_{1}, G_{2}$, or $G_{3}$. But, since $L$ is not a subset of an element of $G_{1}$, an element of $G_{2}$, and an element of $G_{3}$, this is contrary to Theorem 9 .

\section{Examples of a $W_{2}$ set and a $W_{3}$ set.}

THEOREM 11. Every compact continuum whose boundary is the sum of two mutually exclusive simple closed curves is a $W_{n}$ set, for each $n$.

Proof. It follows from the proof of Theorem 2 that if $M^{\prime}$ denotes $C_{2}$ plus its interior, then there exist $n$ collections of arcs, $G_{1}, G_{2}, \cdots, G_{n}$, satisfying with respect to $M^{\prime}$ the conditions of Theorem 2 such that, for each $i,(1)$ if $g$ is an end element of $G_{i}, g \cdot C_{2}$ contains an end point of $g$ and is either the point $(2,0)$ or the point $(2, \pi)$ in the polar coordinate system and (2) if $g$ is a non-end element of $G, g \cdot C_{2}$ is the sum of the end points of $g$ and is the common part of $C_{2}$ and some vertical line.

Let $C$ be an arc on $C_{2}$ which contains neither of the points $(2,0)$ and $(2, \pi)$ and let $C^{\prime}$ be its reflection in the $x$-axis. There is an upper semicontinuous collection $G$ of mutually exclusive point sets filling up $M^{\prime}$ such that each element of $G$ is either a point of $M^{\prime}-\left(C+C^{\prime}\right)$ or the common part of $C+C^{\prime}$ and some vertical line. With respect to its elements $G$ is topologically equivalent to the continuum $M$ of Theorem 1, and arguments similar to those used in the proof of Theorem 2 may now be used to show that with respect to its elements $G$ is a $W_{n}$ set and that $M$ is a $W_{n}$ set. Every compact continuum whose boundary is the sum of two mutually exclusive simple closed curves is topologically equivalent to $M$. Hence every such continuum is a $W_{n}$ set.

THEOREM 12. There exists a $W_{2}$ set which is not a $W_{3}$ set.

Proof. Let $s_{0}$ denote the interval $[0,1]$ on the $x$-axis. Let $\alpha$ denote a sequence whose terms, $p_{1}, p_{2}, \cdots$, are the rational numbers between 0 and 1 . Let $s_{1}, s_{2}, \cdots$ and $m_{1}, m_{2}, \cdots$ be two infinite sequences of straight line intervals converging to $s_{0}$ such that, for each $i, s_{i}$ and $m_{i}$ are above the $x$-axis and parallel to it, each of them has one end point on the line $x=0$ and one on the line $x=1$, and $s_{i}$ is between $m_{i}$ and $m_{i+1}$.

Let $M_{1}, M_{2}, \cdots$ be an infinite sequence of squares with interiors $I_{1}, I_{2}, \cdots$ respectively such that, for each $i$, (1) the diagonals of $M_{i}$ are parallel to the axes, (2) the center of $M_{i}$ is on $s_{i}$ and has abscissa $p_{i}$, and (3) $M_{i}$ does not intersect $m_{i}, m_{i+1}$ or either of the lines $x=0$ or $x=1$ and if $j \neq i$ it does not intersect $M_{j}$ or the line $x=p_{j}$. Let $t_{0}$ and $t_{1}$ denote the intervals of the lines $x=0$ and $x=1$ whose end points lie on $s_{0}$ and $m_{1}$. Let $N$ denote the rectangular disc bounded by $s_{0}+m_{1}+t_{0}+t_{1}$.

Let $K$ be an upper semicontinuous collection of mutually exclusive con- 
tinuous curves which fills up $N-\sum_{i=1}^{\infty} I_{i}$, is an arc with respect to its elements, and is such that its end elements are $t_{0}$ and $t_{1}$, no element of it contains a horizontal interval, and, if $k$ is an element of $K, k \cdot\left(s_{0}+m_{1}\right)$ is the common part of $s_{0}+m_{1}$ and some vertical line. Let $H$ be the collection of all point sets $h$ such that $h$ is the common part of $N-\sum_{i=1}^{\infty} I_{i}$ and some horizontal line.

Let $G$ be an upper semicontinuous collection of mutually exclusive point sets filling up $N-\sum_{i=1}^{\infty} I_{i}$ such that each of its elements is either the common part of $t_{0}+t_{1}$ and some horizontal line between $s_{0}$ and $m_{1}$ or a point of $N-\left(\sum_{i=1}^{\infty} I_{i}+t_{0}+t_{1}\right)$. With respect to its elements $G$ is topologically equivalent to a bounded subset of the plane and the methods of Theorems 2 and 11 can be used to prove that $G$ is a $W_{2}$ set with respect to its elements. However, it follows from Theorem 10 that it is not a $W_{\mathbf{3}}$ set.

The following theorems also hold true.

THEOREM 13. If $M$ is a $W_{3}$ set, $B$ is its boundary, and $J$ is the boundary of a complementary domain of $M$, then there do not exist seven points of $J$ each of which is a limit point of $B-J$.

THEOREM 14. There exists $a W_{3}$ set $M$ with boundary $B$ and a complementary domain of $M$ whose boundary $J$ contains six limit points of $B-J$.

THEOREM 15. If $M$ is a $W_{7}$ set, $B$ is its boundary, and $J$ is the boundary of a complementary domain of $M$, then there do not exist three points of $J$ each of which is a limit point of $B-J$.

7. Concerning $W_{2}^{\prime}$ sets. In what follows, if $M$ is a compact continuum in the plane, $B(M)$ will denote its boundary and $S(M)$ will denote the collection consisting of all boundaries of complementary domains of $M$, all points of $B(M)$ that are not on the boundary of any complementary domain of $M$, and all points of $B(M)$ that are common to the boundaries of two or more complementary domains of $M$. If $S(M)$ is an upper semicontinuous collection of type two, let $Q_{S(M)}$ denote a space whose "points" are the elements of $S(M)$ and whose "regions" are the domains $\left({ }^{9}\right)$ of elements of $S(M)$, the "point" $x$ being "contiguous" to the "point" $y$ if and only if either $x$ is an ordinary point of the continuum $y$ or $y$ is an ordinary point of the continuum $x$.

The following theorem will be proved.

THEOREM 16. The compact continuum $M$ in the plane is a $W^{\prime}$ set if and only if (1) every boundary of a complementary domain of $M$ is a simple closed curve and (2) the collection $S(M)$ is an upper semicontinuous collection of type 2 and there exists an upper semicontinuous collection, $S^{\prime}$, of type 2 such that $S(M)$ is a subcollection of $S^{\prime}$, each element of $S^{\prime}-S(M)$ is a point of $M-B(M), S^{\prime}$ is an

( $\left.{ }^{9}\right)$ R. L. Moore, Fundamental theorems concerning point sets, The Rice Institute Pamphlet, vol. 23, 1936, pp. 43 and 56. 
$\operatorname{arc}\left({ }^{10}\right)$ with respect to its elements, and if $J$ is the boundary of a complementary domain of $M$ there are only two points $P$ of $J$ such that $P$ is a limit point of the sum of the elements of $S^{\prime}$ different from $J$.

Before proceeding to the proof of this theorem, certain lemmas will be established.

Lemma 1. Suppose (1) $M$ is a compact continuous curve in the plane such that every boundary of a complementary domain of $M$ is a simple closed curve, (2) $G$ is a continuous collection of mutually exclusive continuous curves which fills up $M$ and is an arc with respect to its elements, and (3) $g$ is a non-end element of $G$ which intersects the boundary $J$ of some complementary domain of $M$. Then $J \cdot g$ does not contain an arc.

Proof. Suppose $J \cdot g$ does contain an arc, $t$. The point set $M-g$ is the sum of two mutually separated point sets $K$ and $L$. Since $G$ is continuous, $g$ is a subset of the common part of the boundaries of the continuous curves $K+g$ and $L+g$.

Let $P_{1}$ and $P_{2}$ be two cut points of $t$. There are two connected domains with respect to $K+g, D_{1}$ and $D_{2}$, containing $P_{1}$ and $P_{2}$ respectively such that $\bar{D}_{1}$ and $\bar{D}_{2}$ are mutually exclusive and their sum does not contain either end point of $t$. There is an element, $g^{\prime}$, of $G$, different from $g$, but intersecting both $D_{1}$ and $D_{2}$. Some element of $G$ distinct from $g$ contains an arc, $t^{\prime}$, with end points in $D_{1}$ and $D_{2}$ respectively. In $D_{1}$ there is an arc containing $P_{1}$ and some point of $t^{\prime}$ and in $D_{2}$ there is an arc containing $P_{2}$ and some point of $t^{\prime}$. Therefore there is an $\operatorname{arc} P_{1}^{\prime} P_{2}^{\prime}$ lying in $K$ except for its end points, which are cut points of $t$.

Similarly there is an arc $Q_{1}^{\prime} Q_{2}^{\prime}$ lying in $L$ except for its end points, which are separated in $M$ by $P_{1}^{\prime} P_{2}^{\prime}$. Since $K$ and $L$ are mutually separated, this is impossible. Hence $J \cdot g$ does not contain an arc.

Lemma 2. If $J$ is the boundary of a complementary domain of the $W_{2}^{\prime}$ set $M, J$ does not contain three limit points of $B(M)-J$.

Proof. Let $G_{1}$ and $G_{2}$ denote two collections with respect to which $M$ is a $W_{2}^{\prime}$ set such that no element of $G_{1}$ contains $J$. The collection $H$ of all elements of $G_{1}$ that intersect $J$ is an arc with respect to its elements. Let $h_{1}$ and $h_{2}$ be its end elements and let $H_{1}$ denote the collection $H-\left(h_{1}+h_{2}\right)$. Suppose three points, $P_{1}, P_{2}$, and $P_{3}$, of $J$ are limit points of $B(M)-J$. If one of these were a point of $H_{1}^{*}$, there would exist a complementary domain of $M$ whose boundary is a subset of $H_{1}^{*}$ and consequently a subset of some element of $H_{1}$. (See Theorem 8, Corollary.) But, since no element of $H_{1}$ is an end element of $G_{1}$, this is contrary to Lemma 1 . Therefore two of the points $P_{1}, P_{2}$, and $P_{3}-$ say $P_{1}$ and $P_{2}$-are in the same one of the sets $h_{1}$ and $h_{2}$-say $h_{1}$. It follows

(10) R. L. Moore, loc. cit., p. 2. 
that $J \cdot h_{1}$ is an arc. (See Theorem 8.) Hence, by Lemma $1, h_{1}$ is an end element of $G_{1}$.

For $i$ equal to 1 or $2, P_{i}$ is in the sequential limiting set of a sequence $J_{i 1}, J_{i 2}, J_{i 3}, \cdots$ each term of which is the boundary of a complementary domain of $M$ and is different from $J$. Since no element of either of these sequences is a subset of $H_{1}^{*}$, and $h_{1}$ does not separate $M$, there exists a positive number $\delta$ such that if $n$ is greater than $\delta, J_{1 n}$ and $J_{2 n}$ are subsets of $h_{1}$.

Since $J \cdot h_{1}$ contains an arc, no element of $G_{2}$ contains $J$. Hence, for some $m$ greater than $\delta, J_{1 m}$ or $J_{2 m}$ is a subset of the common part of an element of $G_{1}$ and an element of $G_{2}$. This involves a contradiction.

LemMA 3. If $M$ is a $W_{2}^{\prime}$ set and $K_{1}$ and $K_{2}$ are nondegenerate continua such that their common part is the point $P$ and each of them is the sum of some elements( $\left.{ }^{11}\right)$ of $S(M)$, then $P$ is not a limit point of $B(M)-\left(K_{1}+K_{2}\right)$.

Proof. Suppose $P$ is a limit point of $B(M)-\left(K_{1}+K_{2}\right)$. Let $S_{3}$ denote a sequence $J_{31}, J_{32}, \cdots$, having $P$ in its sequential limiting set such that for each $i, J_{3 i}$ intersects $B(M)-\left(K_{1}+K_{2}\right)$ and is the boundary of a complementary domain of $M$. If $j$ is 1 or 2 let $S_{j}$ denote a sequence $J_{j 1}, J_{j 2}, \cdots$, having $P$ in its sequential limiting set such that, for each $i, J_{j i}$ lies in $K_{j}$ and is the boundary of a complementary domain of $M$.

Let $G_{1}$ and $G_{2}$ be two collections with respect to which $M$ is a $W_{2}^{\prime}$ set. Let $g_{1}$ be the element of $G_{1}$ containing $P$. Suppose that a component, $U$, of $M-g_{1}$ has the property that each of two of the sequences $S_{1}, S_{2}$, and $S_{3}$ has a subsequence each element of which intersects $U$ and has $P$ in its sequential limiting set. One of these two sequences is either $S_{1}$ or $S_{2}$. Suppose the two sequences are $S_{1}$ and $S_{3}$. Since $K_{1}$ is a continuum and intersects $U$, there exists a positive integer $n$, a non-end element $g^{\prime}$ of $G_{1}$, and a boundary, $J^{\prime}$, of a complementary domain of $M$ such that $g^{\prime}$ is a subset of $U, J^{\prime}$ is a subset of $K_{1}$, and $J^{\prime} \cdot g^{\prime}$ and $J_{3 n} \cdot g^{\prime}$ exist and are totally disconnected. However, it follows from Theorem 6 that this is impossible. Consequently, for one of the sequences $S_{1}, S_{2}$, and $S_{3}$-say $S_{1}$-there is a positive integer $\delta_{1}$ such that if $n$ is greater than $\delta_{1}, J_{1 n}$ is a subset of $g_{1}$. It follows from Lemma 1 that $g_{1}$ is an end element of $G_{1}$ and consequently that $M-g_{1}$ has only one component. Hence, for one of the sequences $S_{2}$ and $S_{3}$-say $S_{2}$-there is a positive integer $\delta_{2}$ such that if $n$ is greater than $\delta_{2}, J_{2 n}$ is a subset of $g_{1}$.

In a similar manner it may be shown that if $g_{2}$ is the element of $G_{2}$ containing $P$, then for one of the sequences $S_{1}$ and $S_{2}$ - say $S_{1}$ - there is a positive integer $\delta$ greater than $\delta_{1}$ such that if $n$ is greater than $\delta, J_{1 n}$ is a subset of $g_{2}$. Since $J_{1 n}$ is also a subset of $g_{1}$, this is impossible; hence the supposition that $P$ is a limit point of $B(M)-\left(K_{1}+K_{2}\right)$ is false.

(11) It follows from Lemma 1 and Theorem 9 that no nondegenerate continuum of elements of $S(M)$ is made up entirely of elements of $S(M)$ which are ordinary points of $M$. 
Lemma 4. If $M$ is a $W_{2}^{\prime}$ set, there is no simple closed curve in the space $Q_{S(M)}$.

Proof. It follows from Lemmas 1 and 2 and Theorem 9 that $S(M)$ is an upper semicontinuous collection of type 2, so the space $Q_{S(M)}$ exists. Suppose $K$ is a simple closed curve in the space $Q_{S(M)}$. Since no continuum in $Q_{S(M)}$ is the sum of points $\left({ }^{12}\right)$ of $S(M)$ and since $M$ is not separated by any finite point set $\left({ }^{13}\right)$, it follows that infinitely many elements of $K$ are boundaries of complementary domains of $M$.

There exists such a boundary, $J$, and an integer $i$, less than 3 , such that no end element of $G_{i}$ intersects $J$. Let $h_{1}$ and $h_{2}$ denote the end elements of the collection, $H$, of all elements of $G_{i}$ that intersect $J$ and let $H_{1}$ denote $H-\left(h_{1}+h_{2}\right)$. The point set $G_{i}^{*}-H^{*}$ is the sum of two mutually separated connected point sets $L$ and $N$.

Since $K$ is a simple closed curve, it follows from Lemmas 1,2 , and 3 that there exist two points, $P_{1}$ and $P_{2}$, of $J$ such that $K^{*}-\left[J-\left(P_{1}+P_{2}\right)\right]$ is a continuum. Call it $K^{\prime}$. If $P_{1}$ or $P_{2}$ were in $H_{1}^{*}, K \cdot H_{1}^{*}$ would contain the boundary of some complementary domain of $M$, which we have seen to be impossible. Since $h_{1} \cdot J$ and $h_{2} \cdot J$ are connected, they are degenerate. These two points are $P_{1}$ and $P_{2}$. Hence $L$ and $N$ intersect $K^{\prime}$ and therefore so does $H_{1}^{*}$. This, however, is impossible. Hence Lemma 4 is proved.

Proof of Theorem 16. First suppose $M$ is a $W_{2}^{\prime}$ set. Let $Z$ denote the set of all "points" of the space $Q_{S(M)}$. It follows from Lemmas 3 and 4 that every component of $Z$ is an atriodic dendron and therefore an arc or a point. It follows from Lemma 3 that if the component $K$ of $Z$ is an arc and $K^{*}$ contains a limit point of $B(M)-K^{*}$, then that point is a point of an end element of $K$. By Lemma 2, no end element of $K$ contains more than one such point. By Lemma 3, if an end element of $K$ is a simple closed curve, $J$, then no point of $J$ is a limit point both of $K^{*}-J$ and of $B(M)-K^{*}$. By Lemma 3, if the simple closed curve $J$ is a non-end element of the nondegenerate component $K$ of $Z$, then $J$ contains only two limit points of $K^{*}-J$. Furthermore, if $J$ is any nondegenerate element of $S(M), J$ contains no more than two limit points of $B(M)-J$.

If $J$, the boundary of a complementary domain of $M$, contains two limit points of $B(M)-J$, denote these points by $P_{J}$ and $Q_{J}$. If it contains only one limit point of $B(M)-J$, denote it by $P_{J}$ and let $Q_{J}$ be a point of $J$ different from $P_{J}$. If it contains no limit point of $B(M)-J$, let $P_{J}$ and $Q_{J}$ be two points of $J$. There is an upper semicontinuous collection, $H_{J}$, of mutually exclusive point sets filling up $J$ such that (1) $H_{J}$ is an arc with respect to its elements, (2) the end elements of $H_{J}$ are $P_{J}$ and $Q_{J}$, and (3) every non-end element of $H_{J}$ is composed of two points separated in $J$ by $P_{J}+Q_{J}$.

(12) See footnote 11.

(13) R. H. Bing, loc. cit., Theorem 1. 
Let $T_{M}$ be the collection consisting of all the elements of $H_{J}$ for every simple closed curve $J$ that is the boundary of a complementary domain of $M$ and all the points of $M$ which are not contained in an element of $H_{J}$ for any $J$. The collection $T_{M}$ is an upper semicontinuous collection of mutually exclusive point sets which fills up $M$ and is the surface of a sphere with respect to its elements. Let $T_{B(M)}$ denote the collection of all elements of $T_{M}$ which are subsets of $B(M)$. With respect to its elements regarded as points $T_{B(M)}$ is a closed point set each component of which is either an arc or a point and whose components form a contracting sequence $\left({ }^{14}\right)$. Furthermore, if the nondegenerate component, $L$; of the set of all elements ("points") of $T_{B(M)}$ contains a limit "point" of $T_{B(M)}-L$, that "point" is an "end point" of $L$. Therefore $T_{B(M)}$ satisfies the conditions of a theorem( $\left.{ }^{15}\right)$ of R. L. Moore and J. R. Kline and as a result there is an arc $\alpha$ in $T_{M}$ which contains $T_{B(M)}$ and which is such that neither of its end elements belongs to $T_{B(M)}$. Each element of $\alpha$ that is not an element of $T_{B(M)}$ is a point of $M$. Let $S^{\prime}$ be the collection consisting of $S(M)$ plus the collection of all elements of $\alpha$ not in $T_{B(M)}$. This collection satisfies all the conditions required of $S^{\prime}$ in the statement of Theorem 16.

Suppose now that $M$ is a continuum satisfying the conditions of Theorem 16. It will be shown that $M$ is a $W_{2}^{\prime}$ set. It is easily seen that $M$ is a continuous curve and that it is a connected domain plus its boundary. Let $K$ denote the sum of the elements of the collection $S^{\prime}$ and let $K^{\prime}$ denote the sum of the end elements of $S^{\prime}$ and the cut points of $K$.

Let $M^{\prime}, C_{2}, G_{1}$, and $G_{2}$ be as defined in the proof of Theorem 11 . Let $\beta$ be an arc on $C_{2}$ which does not intersect the $x$-axis and let $C$ be a closed subset of $\beta$ such that there is a reversibly continuous transformation $T$ of $C$ into $K^{\prime}$ such that if $A, B$, and $O$ are points of $C$ and $O$ separates $A$ from $B$ in $\beta$, then $T(O)$ separates $T(A)$ from $T(B)$ in $K$. Let $C^{\prime}$ be the reflection of $C$ in the $x$ axis.

Let $G$ be an upper semicontinuous collection of mutually exclusive point sets filling up $M^{\prime}$ such that each element of $G$ is either a point of $M^{\prime}-\left(C+C^{\prime}\right)$ or the common part of $C+C^{\prime}$ and some vertical line. With respect to its elements regarded as points $G$ is topologically equivalent to $M$ and it follows from arguments similar to those used in the proofs of Theorems 2 and 11 that $M$ is a $W_{2}^{\prime}$ set.

In a similar way it may be shown that if $n$ is an integer greater than 1 , then Theorem 16 remains true if in its statement " $W_{2}^{\prime}$ " is replaced by " $W_{n}^{\prime} . "$

The University of Texas, Austin, TeX.

(14) This follows from Lemma and Theorem 9.

(15) R. L. Moore and J. R. Kline, On the most general closed point set through which it is possible to pass a simple continuous arc, Ann. of Math. vol. 20 (1919) pp. 218-223. 